Article

\title{
The Theoretical Highest Frame Rate of Silicon Image Sensors
}

\author{
Takeharu Goji Etoh ${ }^{1,2, *}$, Anh Quang Nguyen ${ }^{2}$, Yoshinari Kamakura ${ }^{1}$, Kazuhiro Shimonomura ${ }^{2}$, \\ Thi Yen Le ${ }^{1}$ and Nobuya Mori ${ }^{1}$ \\ 1 Graduate School of Engineering, Osaka University, 2-1 Yamada-Oka, Suita, Osaka 565-0871, Japan; \\ kamakura@si.eei.eng.osaka-u.ac.jp (Y.K.); lethiyen@si.eei.eng.osaka-u.ac.jp (T.Y.L.); \\ nobuya.mori@eei.eng.osaka-u.ac.jp (N.M.) \\ 2 Graduate School of Science and Engineering, Ritsumeikan University, 1-1-1 Noji-Higashi, Kusatsu, \\ Shiga 525-8577, Japan; gr0220pp@ed.ritsumei.ac.jp (A.Q.N.); skazu@fc.ritsumei.ac.jp (K.S.) \\ * Correspondence: yb6t-etu@asahi-net.or.jp; Tel.: +81-901-901-4429
}

Academic Editor: Vittorio M. N. Passaro

Received: 14 January 2017; Accepted: 24 February 2017; Published: 28 February 2017

\begin{abstract}
The frame rate of the digital high-speed video camera was 2000 frames per second (fps) in 1989, and has been exponentially increasing. A simulation study showed that a silicon image sensor made with a $130 \mathrm{~nm}$ process technology can achieve about $10^{10} \mathrm{fps}$. The frame rate seems to approach the upper bound. Rayleigh proposed an expression on the theoretical spatial resolution limit when the resolution of lenses approached the limit. In this paper, the temporal resolution limit of silicon image sensors was theoretically analyzed. It is revealed that the limit is mainly governed by mixing of charges with different travel times caused by the distribution of penetration depth of light. The derived expression of the limit is extremely simple, yet accurate. For example, the limit for green light of $550 \mathrm{~nm}$ incident to silicon image sensors at $300 \mathrm{~K}$ is 11.1 picoseconds. Therefore, the theoretical highest frame rate is 90.1 Gfps (about $10^{11} \mathrm{fps}$ ).
\end{abstract}

Keywords: high speed; image sensor; frame rate; temporal resolution limit; BSI

\section{Evolution of High-Speed Imaging Devices}

In this section, evolution of high-speed imaging devices is summarized, which suggests the importance of searching for the ultimate high-speed of silicon image sensors.

In this paper, a video camera is defined as a camera capable of capturing 100 or more consecutive images. Then, an image can be replayed at 10 frames per second (fps) for more than $10 \mathrm{~s}$. The time constant of the fundamental saccade motion of human eyeballs is $200 \mathrm{~ms}$ or less [1]. Therefore, image frames replayed at $10 \mathrm{fps}$ look sufficiently smooth. This criterion was discussed in 1994 [2] and in 2003 [3] and widely accepted in the design of burst image sensors for video cameras [4-12]. Cameras and other imaging devices capable of capturing less than 100 consecutive images are referred to as multi-framing cameras or devices [13-23].

The first commercially available digital high-speed video camera was Kodak Ektapro EM, first marketed in 1989 in the USA. The frame rate was $2000 \mathrm{fps}$ for a pixel count of $240 \times 192$ pixels. Etoh developed a faster one in 1991 [4]. The frame rate was $4500 \mathrm{fps}$ for $256 \times 256$ pixels to $40,500 \mathrm{fps}$ for $64 \times 64$ pixels. The camera was marketed as Kodak Ektapro HS4540 and Photron FASTCAM (the first generation).

Since then, the frame rates of digital high-speed cameras have rapidly increased, as shown with blue squares and green circles in Figure $1[4,5,8-13,17,18]$. The blue squares indicate the frame rates achieved by standard high-speed cameras with one silicon image sensor. The green circles indicate the frame rates of cameras with a set of multiple cameras $[7,14]$ and a camera with an image 
sensor with macropixels, each consisting of multiple pixels [23]. The red triangles indicate the frame rates recorded with imaging devices supported by various technologies other than high-speed image sensors $[6,15,16,19,20]$. The size of each plot represents the frame counts. The squares drawn with a blue solid line and a dashed line indicate, respectively, the frame rates of silicon image sensors under process and under design based on simulations.

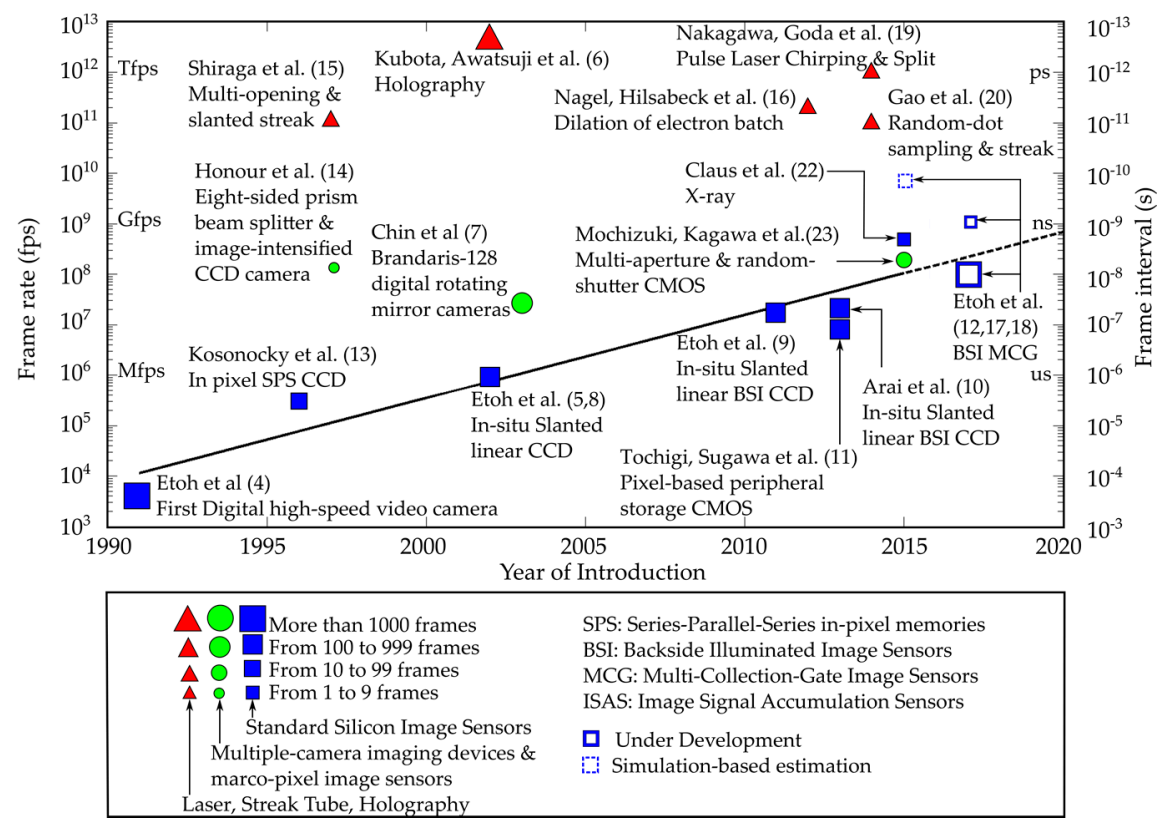

Figure 1. Evolution of high-speed imaging devices.

Cameras with silicon image sensors are compact, sensitive, and user-friendly. Various useful built-in functions can be installed in the sensor. Therefore, the performance is being rapidly improved. For example, the frame rate has exponentially increased (linearly increased in a semilogarithmic scale in Figure 1) from only about $10^{4} \mathrm{fps}$ in the mid-1990s. Test chips of high-speed image sensors for a video camera and a multi-framing camera respectively operating at $10^{8} \mathrm{fps}$ and $10^{9} \mathrm{fps}$ are under process [12]. For X-ray imaging, image sensors capable of capturing 2 or 4 frames at the frame interval of $2 \mathrm{~ns}$ were developed in the image sensor team of Sandia National Laboratories [22]. A simulation study predicted that the shortest frame interval, less than $200 \mathrm{ps}\left(0.5 \times 10^{10} \mathrm{fps}\right)$, can be achieved by a silicon image sensor with a $130 \mathrm{~nm}$ process technology both for the sensor structure and the driving circuit for charge detection [21]. On the other hand, the frame rates of other continuous imaging devices mostly stay between $10^{11} \mathrm{fps}$ and $10^{12} \mathrm{fps}$. The highest frame rate of the cameras with silicon image sensors has come closer to the frame rate achievable only by other high-speed imaging devices in the past.

There is no plot below the trend line indicating the frame rates of the silicon image sensors. Once a silicon image sensor achieves a frame rate, the user-friendliness has eliminated other high-speed imaging devices operating at the frame rate similar to or less than the frame rate of the silicon image sensor. For example, when the digital high-speed video camera of $4500 \mathrm{fps}$ was developed (1991), high-speed film cameras had already achieved a frame rate much higher than 10,000 fps with high-resolution color images. Still, they completely disappeared before 2000. Exploring the ultimate potential of silicon image sensors is essential to forecast the future of the field of high-speed imaging.

The spatial resolution limit was discussed by Abbe, Rayleigh, and others, when the resolution of lenses approached the limit [24]. The frame rate of silicon image sensors seems to approach the upper bound. Research relating to the highest possible frame rate of silicon image sensors has just begun $[21,25,26]$. It is time to search for the temporal resolution limit. 
The highest frame rate of an image senor is limited by either the performance of the photoelectron generation and the transport processes in the photoelectron conversion layer, or that of the detection circuit. The former is the subject of this paper. Fortunately, the temporal resolution limit due to the generation and the transport processes was theoretically derived as described in the following sections.

\section{Assumptions}

\subsection{Sensor Structure for Photo-Conversion}

Assume the following conditions:

(1) The structure of the image sensor is backside-illuminated (BSI).

(2) One incident photon generates one single charge, if the thickness of the sensor is infinite.

(3) Incident photons are perpendicular to the backside surface.

(4) The camera is equipped with a single image sensor.

(5) All pixels are exposed for the same duration to capture one frame.

(6) Each pixel is equipped with one charge detection circuit on the front side.

(7) Signal charges are electrons.

For visible light, these conditions will be satisfied by most cameras in the very near future, except the third one. The expression of the temporal resolution limit derived in Section 3.1 can be easily generalized to light with distributed incident angles, as explained in Section 4.1.

\subsection{Target Wavelengths}

Blue, green, and red rays with the wavelengths of $450 \mathrm{~nm}, 550 \mathrm{~nm}$, and $650 \mathrm{~nm}$ are selected as to be representative of visible light. Table 1 shows their fundamental properties relating to the present analysis.

Table 1. Fundamental properties of light for a silicon crystal [27].

\begin{tabular}{cccc}
\hline $\begin{array}{c}\text { Wavelength } \\
(\mathbf{n m})\end{array}$ & $\begin{array}{c}\text { Photon Energy } \\
\mathbf{( e V )}\end{array}$ & $\begin{array}{c}\text { Absorption Coefficient } \\
\left(\mathbf{c m}^{-\mathbf{1}} \mathbf{)}\right.\end{array}$ & $\begin{array}{c}\text { Penetration Depth } \\
(\boldsymbol{\mu \mathbf { m } )}\end{array}$ \\
\hline 450 & 2.753 & $2.05 \times 10^{4}$ & 0.489 \\
550 & 2.255 & $5.77 \times 10^{4}$ & 1.733 \\
650 & 1.907 & $2.05 \times 10^{3}$ & 4.000 \\
\hline
\end{tabular}

\subsection{Signal Detected by Silicon Image Sensor}

A photon of visible light incident to the backside of an image sensor generates an electron-hole pair. Theoretically, an electromagnetic signal is generated due to photoelectron conversion in the backside layer and propagates at the speed of an electromagnetic wave in the silicon crystal to the front side. Turchetta [25] and Mutoh [26] discussed the temporal resolution from this aspect. However, the signal generated at the photoelectron conversion of visible light is too weak to detect with existing detection circuits. The duration of a photoelectron conversion process is much shorter than a travel time of an electron from the backside to the front side. Therefore, the temporal resolution of image sensors for visible light is governed by motions of charges. The mobility of electrons is higher than that of holes. Hereafter, the temporal resolution is analyzed based on motions of electrons detected at the front side of the sensor.

\subsection{Variation of Travel Time Due to Diffusion and Mixing}

In natural phenomena, dispersion is caused by an integrated effect of diffusion due to random motions and mixing due to other macromotions, where dispersion is defined as the total spreading of the distribution. In most cases, mixing enhances dispersion much more than diffusion. In a pipe flow, 
for example, mixing of slow-speed water near the pipe wall and high-speed water near the center is the major cause of spreading of contaminants in the flow direction.

Figure 2 shows an example cross-section of a pixel of a backside-illuminated image sensor $[17,18,21,28]$. The thickness of the chip is about $30 \mu \mathrm{m}$ to prevent a part of incident light remaining after absorption in the silicon layer from direct intrusion to circuits on the front side. A high bias voltage is applied to the backside to deplete the silicon bulk layer. Figure 3 shows an example of Monte Carlo simulations of the trajectories of signal electrons. The motions of the electrons in layers of each pixel are categorized as follows [21]:

1. In a very thin and very high $\mathrm{p}^{+}$layer near the backside (a hole accumulation layer), S1: the layer is filled with holes to eliminate dark current from the backside interface; a nearly isotropic random motion of electrons causes large diffusion.

2. In a bulk depleted layer, S2: drift of electrons is dominant over the random motion; however, the generation sites spread in the vertical direction due to distribution of the penetration depth of light, which causes distribution of the travel time of the generated signal electrons; mixing of electrons with different travel times results in dispersion in addition to the diffusion due to the random motion.

3. In a layer over a p-type umbrella (a p-well) covering the front-side circuits, S3: the p-well is created to prevent direct intrusion of signal electrons to the circuits; the field is inclined and weak, causing a horizontal drift component with relatively large diffusion; distribution of the horizontal travel distance of a signal electron from the distributed generation site to the center causes mixing of electrons; the mixing with relatively large diffusion results in large dispersion in time.

4. In a hole of the p-well around the center of the pixel, S4: signal electrons move vertically; the dispersion resulting from the random motion associated with the vertical drift is not significant.

5. Under the p-well, S5: the motion of electrons is dependent on the circuit structure around the center on the front side.

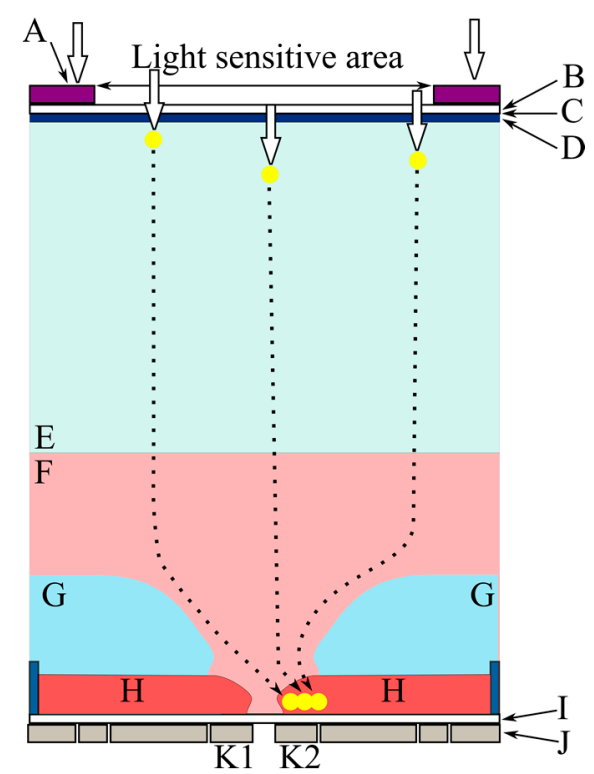

Figure 2. A cross-section structure of a pixel of a backside-illuminated (BSI) image sensor and trajectories of signal electrons. A: light shield; B: oxide; C: interface between the backside oxide layer and the silicon layer; D: backside hole accumulation layer; E: $\mathrm{p}^{-}$layer, F: $\mathrm{n}^{-}$layer; $\mathrm{G}$ : $\mathrm{p}$-well; $\mathrm{H}$ : memory and circuit areas; I: oxide; J: electrodes and wires; K: collection gates. 


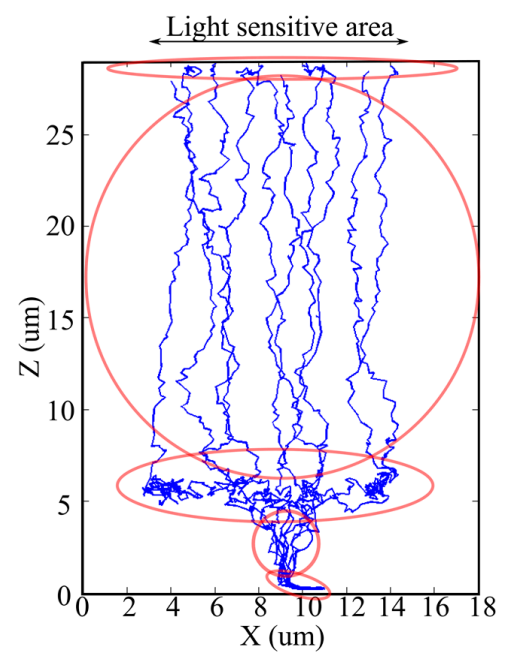

(a)

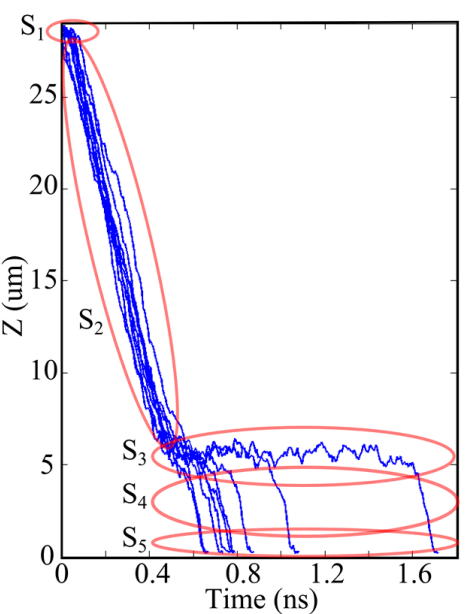

(b)

Figure 3. Monte Carlo simulation of trajectories of signal electrons and segments of the trajectories. (a) Trajectories; (b) travel time versus travel distance from the collection gate. S1: Motion in the backside hole accumulation layer; S2: Motion in the depleted bulk silicon layer; S3: Motion over the p-well; S4: Motion in a p-well hole; S5: Motion to a collection gate.

The hole accumulation layer can be made much thinner than the penetration depth of visible light with an advanced process technology. Then, the whole incident light penetrates the layer and generates electrons in the deeper layer. Therefore, the dispersion in the layer can be neglected in the near future.

The dispersion due to the p-type umbrella can be reduced by design efforts. Figure 4 [21] shows an example. An on-pixel microlens and/or a light guide to focus incident light to the center of each pixel effectively reduces the horizontal mixing. The effect is further enhanced by a light shield with a hole covering the backside.

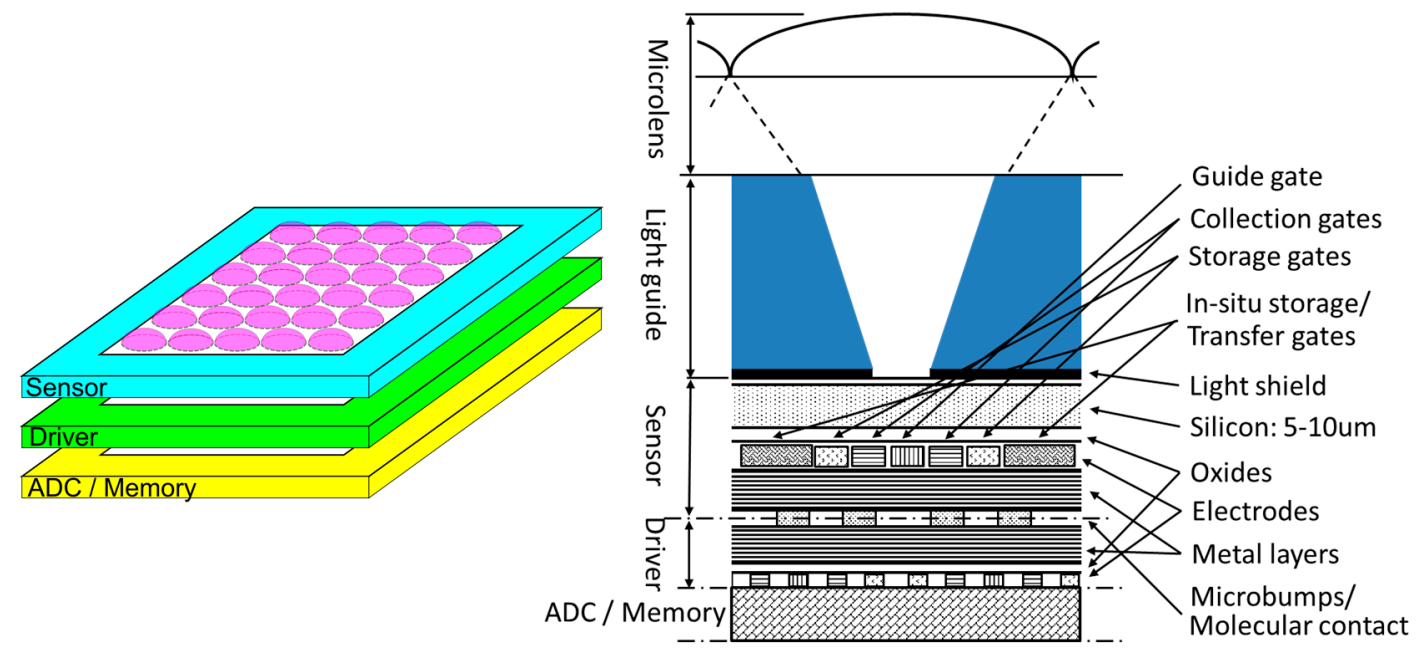

Figure 4. A pixel of a 3D-stacked backside-illuminated multi-collection-gate (BSI-MCG) image sensor with an on-pixel microlens and/or a light guide [21].

On the other hand, the mixing due to the distribution of the penetration depth together with the diffusion due to the random motion cannot be avoided with any design effort. Consequently, these two causes are employed to theoretically derive the temporal resolution limit. 


\subsection{Measure of Temporal Resolution}

The Rayleigh criterion is commonly used as a standard measure of the spatial resolution of optical systems. The criterion is expressed as the distance from the center of the primary Airy disc to the first dark circle. Exactly speaking, the definition does not satisfy the real separation limit, as is well known. The intensity distribution of the superposed two primary Airy discs, according to the Rayleigh criterion, has a dip of $26 \%$ at the center as shown in Figure 5 [29,30]. Dowe's empirical resolution limit prior to Rayleigh's proposal is closer to the no-dip condition, with only a $6 \%$ dip.

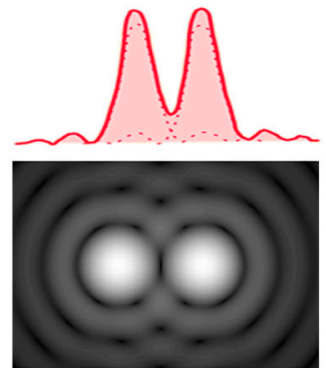

(a)

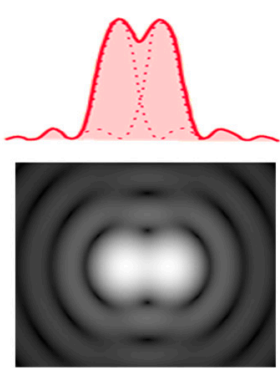

(b)

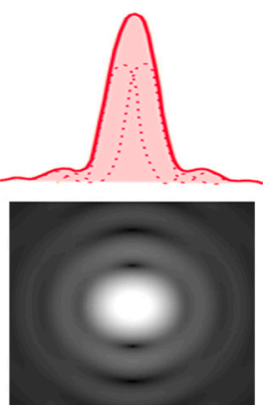

(c)

Figure 5. Airy diffraction patterns generated by light from two points passing through a circular aperture. (a) Points far apart; (b) Rayleigh criterion; (c) points closer than Rayleigh criterion [29,30].

The temporal resolution of imaging devices lowers due to dispersion of arrival times of the signals. Therefore, the standard deviation is the primal measure of the resolution. Different measures are employed for the specific problems, such as a standard imaging and time-stamping. They are all related to the standard deviation. To keep consistency with the measure for the spatial resolution, the no-dip condition expressed by the standard deviation is employed in the present analysis. In the spatial resolution analysis of optical systems, a spot image spreads by diffraction; the shape of the intensity distribution is the primary Airy disc. In the temporal resolution analysis presented later, the travel time spreads by dispersion; the shape of the distribution is Gaussian, except for the distribution observed very close to the generation site.

Assume that two infinitesimal light spots in time and space arrive at the same spot on the backside of the sensor at different times with the interval $\Delta \tau$. A batch of signal electrons generated by each light spot travels to the front side, spreading due to diffusion and mixing. The temporal distribution of the dispersion approaches the Gaussian distribution when the travel distance from the backside increases. During the travel, the difference in the average travel time between two batches of electrons is kept at $\Delta \tau$. The no-dip condition for two superposed Gaussian distributions is strictly $\Delta \tau=2 \sigma$, as shown in Figure 6 , where $\sigma$ is the standard deviation of one Gaussian distribution. Therefore, the value $2 \sigma$ is employed as the measure of the temporal resolution [21].

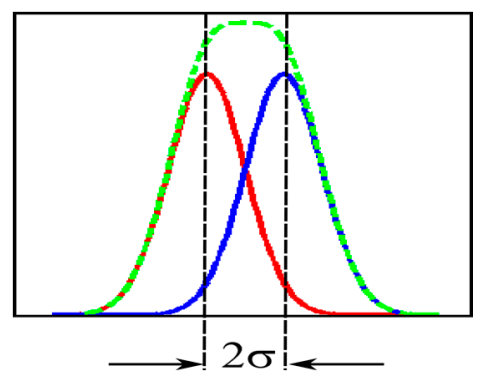

Figure 6. Separation limit (no-dip condition) for two superposed Gaussian distributions; $\sigma:$ standard deviation. 


\subsection{Monte Carlo Simulation Model}

A Monte Carlo simulation model developed by Kunikiyo et al. [31] is employed. The model is based on calculation of a precise band structure of an electron in a silicon crystal. The model automatically includes simulation of phenomena appearing in a higher field, such as impact ionization generating secondary electrons, energy allocation of the impacting electron to the secondary electrons and a phonon. Parameters such as the drift velocity $v$ and the diffusion coefficient $\mathrm{D}$ can be calculated through the simulation. However, the model does not include processes such as a successive impact ionization for a much higher field, recombination of generated electrons and holes, and feedback of the space-field effect of generated electrons and holes.

\subsection{Drift Velocity and Diffusion Coefficient}

Impurity dopants decrease the drift velocity $v$ and increase the diffusion coefficient $\mathrm{D}$, which enhances dispersion. Therefore, an intrinsic silicon layer is assumed to derive the theoretical temporal resolution limit. Figure $7 \mathrm{a}, \mathrm{b}$ respectively show the dependency of $v$ and $\mathrm{D}$ on the field $E$ for the intrinsic silicon at $300 \mathrm{~K}$. The values of $v$ and D calculated by the advanced Monte Carlo simulations are compared with the experimental data [30-32].

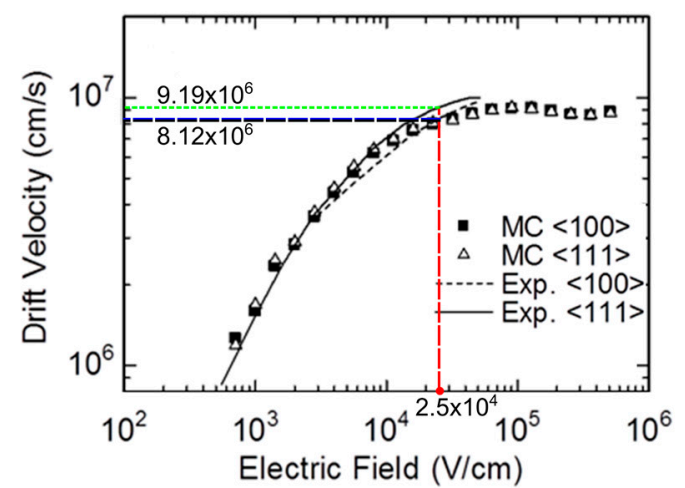

(a)

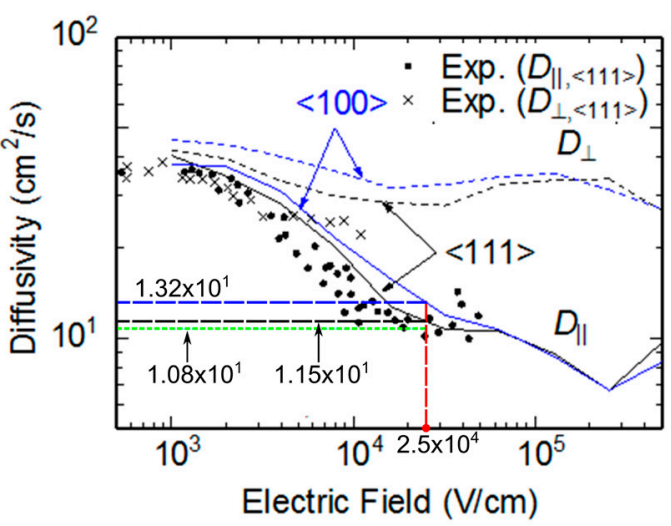

(b)

Figure 7. (a) Drift velocity with respect to electric field at $300 \mathrm{~K}$ [31,32]; (b) diffusivity with respect to electric field at $300 \mathrm{~K}[31,33]$.

For the drift velocity, the results of the Monte Carlo simulation agree well with the experimental data. The drift velocity increases proportionally to the field in a normal operation range of fields, which are less than $1.0 \times 10^{4} \mathrm{~V} / \mathrm{cm}$, and approaches a constant value at the field around $2.5 \times 10^{4} \mathrm{~V} / \mathrm{cm}$ due to frequent scattering events by higher-energy electrons colliding with the silicon lattice. Hereafter, the value of the field for the saturated drift velocity, $2.5 \times 10^{4} \mathrm{~V} / \mathrm{cm}$, is referred to as a critical field.

The Monte Carlo simulation model can separately provide the drift velocities and the diffusion coefficients in the directions parallel to and perpendicular to the travel direction and for different crystal orientations. The simulated diffusion coefficients are slightly higher than the experimental data. Another important discrepancy between the simulated and experimental data appear at the field higher than the critical field. The vertical diffusion coefficient $\mathrm{D}$ parallel to the travel direction reduces along the field until the critical field. The experimental data seem to take a minimum at the critical field. On the other hand, the Monte Carlo simulation results (curves) further decrease for the higher field. The discrepancy may be due to the insufficient expression for fundamental processes for the very high field in the Monte Carlo simulation model explained in Section 2.6. Naturally, the cascade generation process of the secondary electrons which appear in a very high field may increase the diffusion coefficient again. 
The drift velocity and the diffusion coefficient for the crystal orientation $<111>$ are higher and lower, respectively, than those for $<100>$. Therefore, the values for $<111>$ are employed to calculate the values of the temporal resolution limit.

\section{Expression of Standard Deviation of Travel Time}

\subsection{Derivation}

The structure of a BSI image sensor is simplified to include the factors unavoidable and essential for the analysis of the temporal resolution limit, as shown in Figure 8. The model includes the distribution of the penetration depth of photons and the random motion of signal electrons. A constant field is created by a backside bias voltage.

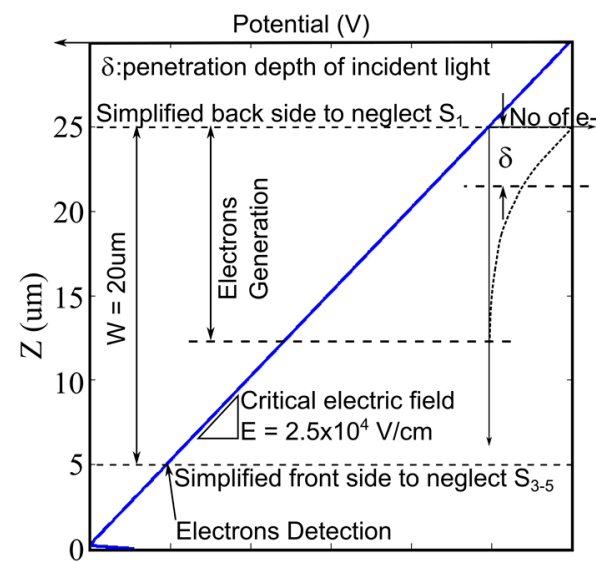

(a)

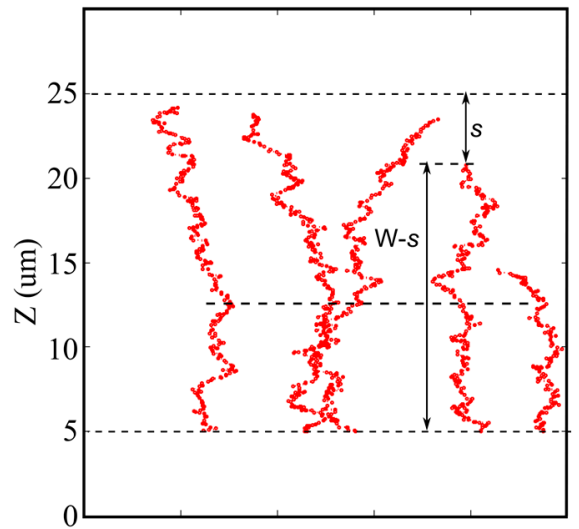

(b)

Figure 8. A cross-section structure for derivation of the standard deviation $\sigma$ of the travel time for $\mathrm{W}=20 \mu \mathrm{m}$. (a) The model for the analysis; (b) example trajectories of electrons.

The travel distance of an electron generated at the depth $s$ from the backside to the front side is $(\mathrm{W}-s)$, where $\mathrm{W}$ is the thickness of the chip. The average travel time $t_{r}$ is $(\mathrm{W}-s) / v$. The probability density function $\mathrm{f}(s)$ of the penetration depth $s$ is as follows:

$$
\mathrm{f}(s)=(1 / \delta) \exp (-s / \delta)
$$

where $\delta$ is the average penetration depth.

The zeroth to the second moments of the average travel time around the origin are:

$$
\begin{gathered}
p=\int_{0}^{\mathrm{W}} \mathrm{f}(s) \mathrm{d} s=1-\exp (-\mathrm{W} / \delta) \\
\mathrm{E}\left(t_{r}\right)=\int_{0}^{\mathrm{W}}\{(\mathrm{W}-s) / v\}(\mathrm{f}(s) / p) \mathrm{ds}=(\mathrm{W}-\delta p) /(v p) \\
\mathrm{E}\left(t_{r}{ }^{2}\right)=\int_{0}^{\mathrm{W}}\{(\mathrm{W}-s) / v\}^{2}(\mathrm{f}(s) / p) \mathrm{d} s \\
=\left(\mathrm{W}^{2}-2 \mathrm{~W} \delta+2 \delta^{2}\right) / v^{2}+\frac{\mathrm{W}^{2}-2 \mathrm{~W} \delta}{v^{2} p} \exp (-\mathrm{W} / \delta)
\end{gathered}
$$

Then, the variance $\sigma_{m}^{2}$ due to the mixing effect of electrons penetrating to different depths is:

$$
\sigma_{m}^{2}=\mathrm{E}\left(t_{r}^{2}\right)-\left\{\mathrm{E}\left(t_{r}\right)\right\}^{2}
$$


After some cumbersome manipulation of equations, the expression for $\sigma_{m}{ }^{2}$ was fortunately reduced to a very simple form:

$$
\sigma_{m}^{2}=\frac{\mathrm{W}^{2}}{p^{2} v^{2}}\left[-\exp (-\mathrm{W} / \delta)+\frac{\delta^{2}}{\mathrm{~W}^{2}} p^{2}\right]=-\frac{\delta^{2} \mathrm{~W}^{\prime 2}}{p^{2} v^{2}} \exp \left(-\mathrm{W}^{\prime}\right)+\frac{\delta^{2}}{v^{2}}
$$

where $\mathrm{W}^{\prime}=\mathrm{W} / \delta$.

Random motion of signal electrons is superposed on the drift motion. The solution of the drift and diffusion equation shows that, when the travel time increases, the travel time distribution approaches the Gaussian distribution [34]. The average travel time is $t_{r}$ and the variance is $2 \mathrm{D} / v^{2}$. Therefore, the variance is proportional to $t_{r}$ multiplied by $2 \mathrm{D} / v^{2}$. The average travel time $t_{r}$ distributes due to the distribution of the penetration depth of light. Therefore, the variance $\sigma_{\mathrm{d}}{ }^{2}$ due to the diffusion effect is given by integration of the variance conditional to the average travel time weighted by the distribution of the penetration depth. Then,

$$
\sigma_{d}^{2}=\frac{2 \mathrm{D}}{v^{2}} \mathrm{E}\left(t_{r}\right)=\frac{2 \mathrm{D}}{v^{2}} \int_{0}^{\mathrm{W}}\{(\mathrm{W}-s) / v\}(\mathrm{f}(s) / p) \mathrm{ds}=\frac{\left(\mathrm{W}^{\prime}-p\right)}{p} \frac{2 \mathrm{D}}{v^{2}} \frac{\delta}{v}
$$

The mixing due to the distribution of the penetration depth and the diffusion conditioned by the average travel time are independent processes. Therefore, the total variance $\sigma_{s}{ }^{2}$ is the sum of $\sigma_{m}{ }^{2}$ and $\sigma_{d}^{2}$.

$$
\sigma_{s}^{2}=\sigma_{m}^{2}+\sigma_{d}^{2}
$$

Then, the temporal resolution limit $\Delta \tau$ is

$$
\Delta \tau=2 \sigma_{s}
$$

Equation (9) is generally applicable to photo-charge conversion layers, which satisfy the conditions stated in Section 2.1. The value is calculated by specifying the values of the parameters included in the expression: the drift velocity, the diffusion coefficient, and the thickness of the layer.

Sensitivity is crucial in ultrahigh-speed imaging. The quantum efficiency seriously decreases for the thickness less than the average penetration depth. The temporal resolution limit becomes larger for an increase of the thickness as shown in Equations (6)-(9). Therefore, the thickness can be safely assumed to be equal to the average penetration depth (i.e., $\mathrm{W}^{\prime}=\mathrm{W} / \delta=1$ ).

Then, $p=1-e^{-1}=0.6321$, and the temporal resolution limit is:

$$
\Delta \tau=2 \sqrt{0.079 \frac{\delta^{2}}{v^{2}}+0.582 \frac{2 \mathrm{D}}{v^{2}} \frac{\delta}{v}}
$$

Later, Monte Carlo simulations for silicon image sensors show that $\sigma_{m} \gg \sigma_{d}$. Then, the diffusion effect $\sigma_{d}$ (the second term) is neglected. The value of the drift velocity $v$ for the experimental data in Figure $7 \mathrm{a}$ is $9.19 \times 10^{-2} \mu \mathrm{m} / \mathrm{ps}$. Then, the expression of the temporal resolution limit is reduced to an extremely simple form:

$$
\Delta \tau=0.562 \frac{\delta}{v}=6.12 \delta
$$

where the units of $\Delta \tau$ and $\delta$ are, respectively, ps and $\mu \mathrm{m}$.

It is proved in Section 3.2 that the accuracy of Equation (11) is sufficiently high.

\subsection{Comparison with Monte Carlo Simulation}

Equation (9) is compared with results of Monte Carlo simulations for an intrinsic silicon layer. The diffusion coefficient estimated by the Monte Carlo simulations is slightly higher than the experimental data, as shown in Figure $7 \mathrm{~b}$. Therefore, in comparing the derived expression of the 
temporal resolution limit with the result of the Monte Carlo simulations, the values of $v$ and D estimated through the Monte Carlo simulations are used. To finally estimate the temporal resolution limit later, the values estimated from the experimental data are substituted into the derived expression (9).

For the critical field $2.5 \times 10^{4} \mathrm{~V} / \mathrm{cm}$ at $300 \mathrm{~K}$, the drift velocity and the diffusion coefficient for the crystal orientation $<111>$ are, respectively, $8.12 \times 10^{6} \mathrm{~cm} / \mathrm{s}$ and $11.5 \mathrm{~cm}^{2} / \mathrm{s}$.

Example results are shown in Figure 9 for blue, green, and red light of $450 \mathrm{~nm}, 550 \mathrm{~nm}$, and $650 \mathrm{~nm}$. The solid lines and the dots respectively indicate the temporal resolution $2 \sigma_{\mathrm{s}}$ calculated from Equation (9) and by the Monte Carlo simulations. They agree almost perfectly for a thickness larger than $1 \mu \mathrm{m}\left(10^{-4} \mathrm{~cm}\right)$. The agreement is confirmed for the field range of $0.5 \times 10^{4} \mathrm{~V} / \mathrm{cm}$ to $2.5 \times 10^{4} \mathrm{~V} / \mathrm{cm}$, though the results are not shown.

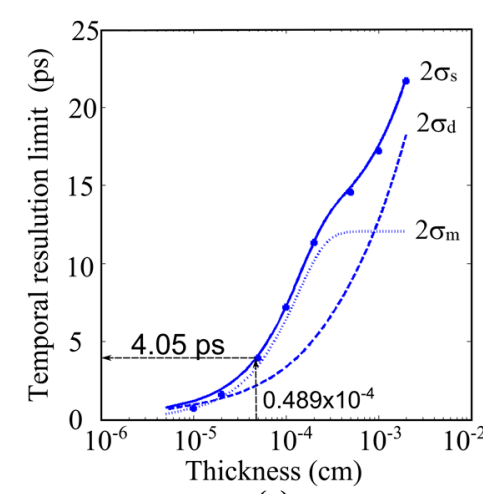

(a)

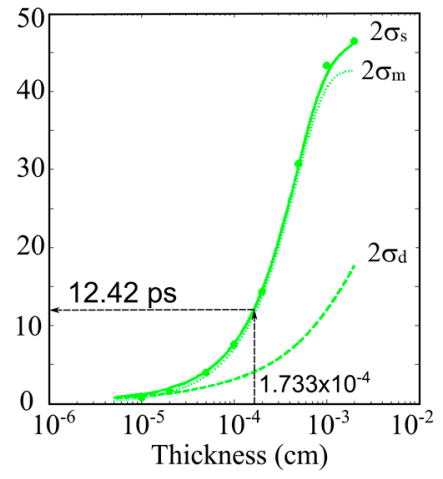

(b)

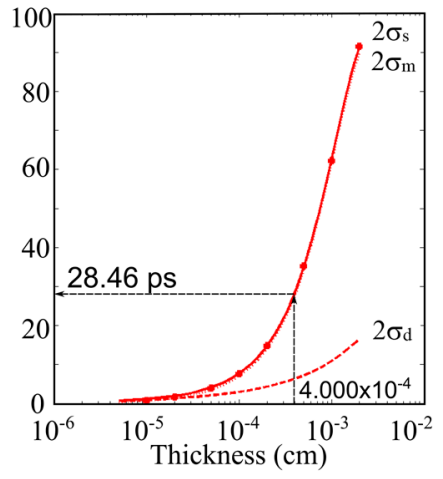

(c)

Figure 9. Proof of the derived expression of the temporal resolution limit. (Solid lines: the temporal resolution limit $2 \sigma_{s}$, Equation (9); dotted lines: the mixing effect $2 \sigma_{m}$, Equation (6); dashed lines: the diffusion effect $2 \sigma_{d}$, Equation (7); dots: Monte Carlo simulations.) (Intrinsic silicon <111>; $300 \mathrm{~K}$; electric field: $2.5 \times 10^{4} \mathrm{~V} / \mathrm{cm}$; wavelength: (a) $450 \mathrm{~nm}$; (b) $550 \mathrm{~nm}$; (c) $650 \mathrm{~nm}$.)

The dashed lines and the dotted lines further show the mixing effect $2 \sigma_{m}$ and the pure diffusion effect $2 \sigma_{d}$. They show the following characteristics:

(1) For a thickness more than $1 \mu \mathrm{m}$, the agreement is almost perfect. For a thickness less than $0.5 \mu \mathrm{m}$, the theoretical value is slightly larger than the result of the Monte Carlo simulation. The reason is the large skew of the distribution of the travel time observed near the generation site.

(2) The temporal resolution of blue light of $450 \mathrm{~nm}$ shows a strange curve. The reason is that the curve expresses a compound effect of two comparable and completely different effects due to the pure diffusion and the mixing. For a thickness less than $3 \mu \mathrm{m}$, the mixing effect is dominant; for a thickness larger than $3 \mu \mathrm{m}$, the mixing effect saturates and the diffusion effect becomes dominant.

(3) For green and red light, the mixing effect due to the distribution of the penetration depth is dominant, and the diffusion effect is practically negligible.

The average penetration depth $\delta$ is shown in Table 1. For example, for the green light, $\delta=1.733 \mu \mathrm{m}$. Then, the temporal resolution limit $\Delta \tau$ is $12.4 \mathrm{ps}$ from the figure or Equation (10). Similarly, for blue and red light, $\Delta \tau$ is 4.05 ps and 28.5 ps, respectively.

An approximate value of the temporal resolution limit $\Delta \tau$ is calculated with Equation (11) as $12.0 \mathrm{ps}$, which is sufficiently close to 12.4 ps obtained from Equation (10).

When the experimental data shown in Figures 4 and 5 for the critical field are employed, instead of the values calculated by Monte Carlo simulations, the drift velocity is $9.19 \times 10^{6} \mathrm{~cm} / \mathrm{s}$ and the diffusion coefficient is $10.8 \mathrm{~cm}^{2} / \mathrm{s}$. Then, the temporal resolution limit for green light is $11.1 \mathrm{ps}$ for Equation (10). Therefore, the upper-bound frame rate is 90.1 Gfps.

If we use Equation (11), $\Delta \tau=10.7$ ps (error: $4 \%$ ), in spite of the extremely simple expression. For the blue and the red light, the errors are $14 \%$ and negligible. 


\subsection{Summary of Logic}

The temporal resolution limit of photo-charge conversion layers is theoretically derived. It is expressed with an extremely simple expression. Since the derivation is complicated with several logical steps, the flow is summarized as explained below ((i)-(vii)). As an example, the temporal resolution limit of silicon image sensors is estimated from the expression and the values of the electronic parameters of silicon crystals ((viii)-(v)).

(i) The resolution is limited by the distribution of travel times of signal charges.

(ii) There are two unavoidable factors affecting the distribution: (a) mixing of signal electrons with different travel distances due to the distributed penetration depths of photons; (b) pure diffusion due to random motions of electrons.

(iii) The expressions of variances $\sigma_{\mathrm{m}}{ }^{2}$ and $\sigma_{\mathrm{d}}{ }^{2}$ of the travel time caused by the mixing and the diffusion are separately derived and added, since they are independent processes.

(iv) The no-dip condition for two superposed Gaussian distributions is employed as the temporal separation limit of batches of electrons generated by two photon groups each instantly arriving at the layer with a time difference $\Delta \tau$, where the no-dip condition is $2 \sigma$, and $\sigma$ is the standard deviation of one Gaussian distribution (Equation (9)).

(v) The derived expression includes drift of the velocity $v$, the diffusion coefficient $D$, and the average penetration depth $\delta$. The temporal resolution limit $2 \sigma$ is calculated by substituting the values of $v$, $\mathrm{D}$, and $\delta$ into Equation (9).

(vi) Equation (9) is verified by the comparison with the results of the Monte Carlo simulations for the values of $v$ and $\mathrm{D}$ for the critical field.

(vii) The thickness $\mathrm{W}$ is assumed to be equal to the average penetration depth $\delta$ to prevent the sensitivity from decreasing for $\delta<\mathrm{W}$, and the temporal resolution from increasing for $\delta>\mathrm{W}$. Therefore, $\mathrm{W}^{\prime}=\mathrm{W} / \delta=1$, which provides Equation (10).

(viii) For silicon layers, the temporal resolution $2 \sigma$ is minimized by the values of $v$ and $\mathrm{D}$ at the critical field, $2.5 \times 10^{4} \mathrm{~V} / \mathrm{cm}$.

(ix) For example, for green light of $550 \mathrm{~nm}$, the temporal resolution limit is 11.1 ps for Equation (10), which is equivalent to $90.1 \mathrm{Gfps}$.

(x) Later, Monte Carlo simulations show $\sigma_{m}>>\sigma_{d}$ for the silicon image sensors for ranges of $\mathrm{W}$ in practical applications. Therefore, if the diffusion effect $\sigma_{d}$ is neglected and the value of $v$ for the critical field is inserted, the expression of the temporal resolution limit is finally reduced to an extremely simple expression in Equation (11). The error to the value calculated from Equation (10) is only $4 \%$ for green light.

The derivation is based on some assumptions. For example, the arrival time distribution is expressed as the flux distribution in time passing through a detection plane, which differs from Gaussian distribution when the travel time is short. Though it is possible to formulate the expression of the temporal resolution limit by using the precise expression of the temporal flux distribution, the result is expressed as an implicit equation and requires numerical calculations to see the characteristics of the solution. The derived expression in this paper is explicit and theoretically sound, from which the fundamental characteristics are clearly observed.

\section{Generalization of Present Theory}

\subsection{Distributed Incident Angles of Light}

The average penetration depth $\delta$ in Equation (9) is replaced with a standard deviation $\sigma_{v}$ of vertical components of penetration depths of visible rays with distributed incident angles. The accuracy of the expression will be sufficiently high in practice. 


\subsection{Cooled Sensor}

Cooling is a very important technology for scientific image sensors to decrease dark current noise and decrease cross-talks due to diffusion of signal charges. It is expected that cooling the sensor may improve the temporal resolution. However, Equation (11) implies that the temperature dependency is not significant. At lower temperatures, both the drift velocity $v$ and the average penetration depth $\delta$ increase together and compensate each other's effects. For example, at about $170 \mathrm{~K}$, the values of $v$ and $\delta$ increase by about $40 \%$ [32] and 60\% [35], respectively, compared with those at $300 \mathrm{~K}$. Then, the temporal resolution limit even increases by $14 \%(1.6 / 1.4=1.14)$.

\subsection{X-ray}

The analytical framework presented in this paper can be modified for analyses of the temporal resolution for more generalized cases. For example, for a soft X-ray, Landau distribution of the number of charges suddenly generated by a single photon and Gaussian distribution of the initial spread of the batch of the charges should be incorporated into the present analysis. Since these processes are independent of the mixing and the diffusion processes, the variance of the arrival time due to these effects may be simply added to Equation (8).

In this case, the arrival time is generally measured as the time when the number of charges generated by a single photon and detected by a detection circuit reaches a specified value. The time is also distributed due to the generation and the transportation processes in the photo-charge conversion layer and temporal fluctuations of the detection circuit elements. The latter effect on the temporal resolution has been analyzed in the field. When the detection circuit is improved, the former one will become the target of the research.

\subsection{Macropixel Image Sensor}

An image sensor with macropixels each consisting of several neighboring pixels is referred to as a macropixel image sensor. If each pixel in a macropixel receives photons at different times and the temporal resolution of the detection circuit of each pixel is infinitesimal, the temporal resolution limit of the macropixel image sensor is much less than the temporal resolution limit expressed by Equation (9).

Assume a macropixel image sensor with macropixels each consisting of two pixels. Batches of charges generated by two photon groups incident to the two elemental pixels are not mixed and separately detected, and the average travel times are independently calculated. The variance of the average travel time is proportional to the variance $\sigma_{\mathrm{s}}{ }^{2}$ expressed by Equation (8) divided by the number of charges $N$. The statistical significance of the difference between the average travel times of two batches is tested with the t-test. Therefore, the temporal resolution limit may be defined by the difference corresponding to a given significance level. For $N>30$, the $\mathrm{t}$-distribution can be approximated by a Gaussian distribution. Therefore, if the significance level is given, the temporal resolution limit is easily calculated.

If the target event is reproducible, the temporal resolution can be improved by repeating the imaging. If the number of repetitions is $\mathrm{M}$, the variance is further reduced to $1 / \mathrm{M}$. The result correctly holds for a macropixel image sensor with more elemental pixels, and for imaging devices consisting of more than two cameras.

The number of consecutive images of a macropixel image sensor is the number of the elemental pixels in each macropixel. A disadvantage of the macropixel is reduced sensitivity, inversely proportional to the number of consecutive frames. Loss of sensitivity is crucial in ultrahigh-speed imaging.

If different color filters are attached to $K$ elemental pixels of each macropixel, and lasers with wavelengths corresponding to the color filters are applied at a very short interval for illumination, $K$ consecutive images are captured at the short frame interval. The wavelength of a laser can be swept at a very high speed. Chirping can be used to sweep the wavelength at an extremely high 
speed. This kind of ultrahigh-speed imaging technologies is sometimes called hyperspectral methods. The authors call them time-spectrum conversion methods. The most advanced one of such methods developed by Goda et al. [19].

With time-space conversion methods, image information of different instants is recorded at different positions. The positions are changed at a very high speed. The position-changing methods include a rotating mirror for shooting images on a circular surface (a rotating mirror camera) [7], deflection electrodes to streak electron beams (a streak tube) [14], even a flying light beam [6], and so on.

Random sampling in space or time with advanced signal process technologies are also applied in ultrahigh-speed imaging to distinguish image information captured at different instances.

Most of these technologies utilize image sensors as the final recording devices of image information. The discussion on the temporal resolution of the macropixel image sensor can be directly applied for analysis of the temporal resolution of the image sensors for these ultrahigh-speed imaging technologies. However, the temporal resolution of macropixel image sensors and independently operated multiple standard image sensors in these applications is usually much shorter than the temporal resolutions of other elements of the devices. Therefore, the overall temporal resolution is limited by a critical element of each imaging device or system other than the image sensors.

\section{Conclusions}

The temporal resolution limit of silicon image sensors is theoretically derived. The limit is mainly governed by mixing of charges with different travel times caused by the distribution of penetration depth of light. The final expression is $\Delta \tau=6.12 \delta$, which may be unbelievably simple, but sufficiently accurate. Now, the target is clear. It is time to give it a try to break it.

Acknowledgments: The authors would like to express their appreciation to financial supports of JST-A STEP Grant Number AS2526901J and a part of JSPS KAKENHI Grant Number 16H04358.

Author Contributions: Takeharu Goji Etoh organized the research team and derived the expression. Anh Quang Nguyen and Thi Yen Le confirmed it with numerical simulations guided by Yoshinari Kamakura who developed the Monte Carlo simulation code. Kazuhiro Shimonomura analyzed factors relating to the temporal resolution. Nobuya Mori supervised the research.

Conflicts of Interest: The authors declare no conflict of interest.

\section{References}

1. Wikipedia: Saccade (Timing and Kinematics). Available online: https://en.wikipedia.org/wiki/Saccade (accessed on 5 January 2017).

2. Etoh, T.G.; Takehara, K. Needs, requirements, and new proposals for ultra-high-speed video cameras in Japan. Proc. SPIE 1995, 2513. [CrossRef]

3. Takano, Y.; Etoh, T.G.; Takehara, K. Users' requirements and specification on high-speed video cameras. J. Visual. Soc. Jpn. 2003, 23, 11-14. (In Japanese) [CrossRef]

4. Etoh, T.G. High-speed video camera operating at $4500 \mathrm{fps}$. J. Inst. Telev. Eng. Jpn. 1992, 46, 543-545. (In Japanese)

5. Etoh, T.G.; Poggemann, D.; Ruckelshausen, A.; Theuwissen, A.; Kreider, G.; Folkerts, O.-H.; Mutoh, H.; Kondo, Y.; Maruno, H.; Takubo, K.; et al. A CCD image sensor of 1 Mframes/s for continuous image capturing of 103 frames. In Proceedings of the 2002 IEEE International Solid-State Circuits Conference, (ISSCC 2002), Digest of Technical Papers, San Francisco, CA, USA, 3-7 February 2002; pp. 46-47.

6. Kubota, T.; Awatsuji, Y. Observation of light propagation by holography with a picosecond pulsed laser. Opt. Lett. 2002, 27, 815-817. [CrossRef] [PubMed]

7. Chin, C.T.; Lancee, C.; Borsboom, J.; Mastik, F.; Frijlink, M.E.; Jong, N.; Versluis, M.; Lohse, D. Brandaris 128: A digital 25 million frames per second camera with 128 highly sensitive frames. Rev. Sci. Instrum. 2003, 74, 5026-5034. [CrossRef]

8. Sandhu, A. Ultrafast Imagination. Nat. Photonics 2007, 1, 638. 
9. Etoh, T.G.; Nguyen, D.H.; Dao, V.T.S.; Vo Le, C.; Tanaka, M.; Takehara, K.; Okinaka, T.; van Kuijk, H.; Klaasens, W.; Bosiers, J. A 16 Mfps 165 kpixel backside-illuminated CCD. In Proceedings of the IEEE International Solid-State Circuits Conference (ISSCC 2011), Digest of Technical Papers, San Francisco, CA, USA, 20-24 February 2011; pp. 406-407.

10. Arai, T.; Yonai, J.; Hayashida, T.; Ohtake, H.; van Kuijk, H.; Etoh, T.G. Back-Side-Illuminated image sensor with burst capturing speed of 5.2 Tpixels per second. Proc. SPIE 2013, 8659. [CrossRef]

11. Tochigi, Y.; Hanzawa, K.; Kato, Y.; Kuroda, R.; Mutoh, H.; Hirose, R.; Tominaga, H.; Takubo, K.; Kondo, Y.; Sugawa, S. A global-shutter CMOS image sensor with readout speed of $1 \mathrm{Tpixel} / \mathrm{s}$ burst and $780 \mathrm{Mpixel} / \mathrm{s}$ continuous. IEEE J. Solid State Circuits 2013, 48, 329-338. [CrossRef]

12. Etoh, T.G.; Dao, V.T.S.; Nguyen, A.Q.; Hayahsi, N. Design of an Image Signal Accumulation Sensor with 1120 frames operating at 100 Mfps. In Proceedings of the 15th International Conference on Advanced Technology in Experimental Mechanics, Toyohashi, Japan, 4-8 October 2015.

13. Kosonocky, W.F.; Guand, Y.; Chao, Y.; Kabra, R.K.; Xie, L.; Lawrence, J.L.; Mastrocolla, V.; Shallcross, F.V.; Patel, V. 360/spl times/360-element very-high-frame-rate burst image sensor. In Proceedings of the International Solid-State Circuits Conference, San Francisco, CA, USA, 8-10 February 1996; pp. 182-183.

14. Honour, J. Electronic camera for simultaneous framing and streak recording. Proc. SPIE 1997, 2869. [CrossRef]

15. Shiraga, H.; Miyanaga, N.; Heya, M.; Nakasuji, M.; Aoki, Y.; Azechi, H.; Yamanaka, T.; Mima, K. Ultrafast two-dimensional $\mathrm{X}$-ray imaging with $\mathrm{X}$-ray streak cameras for fusion research (invited). Rev. Sci. Instum. 1997, 68, 745-749. [CrossRef]

16. Nagel, S.R.; Hilsabeck, T.J.; Bell, P.M.; Bradley, D.K.; Ayers, M.J.; Barrios, M.A.; Felker, B.; Smith, R.F.; Collins, G.W.; Jones, O.S.; et al. Dilation X-ray imager a new/faster gated X-ray imager for the NIF. Rev. Sci. Instrum. 2012, 83, 10E116. [CrossRef] [PubMed]

17. Etoh, T.G.; Dao, V.T.S.; Yamada, T.; Charbon, E. Toward One Giga Frames per Second_Evolution of in-Situ Storage Image Sensors. Sensors 2013, 13, 4640-4658. [CrossRef] [PubMed]

18. Etoh, T.G.; Dao, V.T.S.; Shimonomura, K.; Charbon, E.; Zhang, C.; Kamakura, Y.; Matsuoka, T. Toward 1Gfps: Evolution of Ultra-high-speed Image Sensors-ISIS, BSI, Multi-Collection Gates, and 3D-stacking. In Proceedings of the International Electric Devices Meeting, San Francisco, CA, USA, 15-17 December 2014.

19. Nakagawa, K.; Iwasaki, A.; Oishi, Y.; Hirosaki, R.; Tsukamoto, A.; Hirosawa, K.; Liao, H.; Ushida, T.; Goda, K.; Kannari, K.; et al. Sequentially timed all-optical mapping photography (STAMP). Nat. Photonics 2014, 8, 695-700. [CrossRef]

20. Gao, L.; Liang, J.; Li, C.; Wang, L.V. Single-shot compressed ultrafast photography at one hundred billion frames per second. Nature 2014, 516, 74-77. [CrossRef] [PubMed]

21. Dao, V.T.S.; Etoh, T.G.; Shimonomura, K.; Nguyen, A.Q.; Hayashi, N.; Kamakura, Y.; Zhang, C.; Charbon, E.; Goetschalckx, P.; Haspeslagh, L.; et al. Toward 10 Gfps: Factors limiting the Frame Rate of the BSI MCG Image Sensor. In Proceedings of the International Image Sensor Workshop, Vaals, The Netherlands, 8-11 June 2015.

22. Claus, L.; Fang, L.; Kay, R.; Kimmel, M.; Long, J.; Robertson, G.; Sanchez, M.; Stahoviak, J.; Trotter, D.; Porter, J.L. An overview of the Ultrafast X-ray Imager (UXI) program at Sandia Labs. Proc. SPIE 2015, 9591. [CrossRef]

23. Mochizuki, F.; Kagawa, K.; Okihara, S.I.; Seo, M.W.; Zhang, B.; Takasawa, T.; Yasutomi, K.; Kawahito, S. Single-shot 200Mfps 5x3-aperture compressive CMOS imager. In Proceedings of the 2015 IEEE International Solid-State Circuits Conference, (ISSCC 2015), Digest of Technical Papers, San Francisco, CA, USA, 22-26 February 2015; pp. 116-117.

24. Hornak, J. Encyclopedia of Imaging Science and Technology, 1st ed.; Wiley \& Sons: New York, NY, USA, 2002; pp. 1106-1141.

25. Turchetta, R. Notes about the limits of ultra-high speed solid-state imagers. In Proceedings of the International Image Sensor Workshop, Vaals, The Netherlands, 8-11 June 2015.

26. Mutoh, H. Device Simulations for Ultrahigh-Speed and High-Voltage Image Sensors. IEEE Trans. Electron. Devices 2016, 63, 49-56. [CrossRef]

27. Jones, M.H.; Jones, S.H. Optical Properties of Silicon, Virginia Semiconductor, Inc. Available online: http: //www.univie.ac.at/photovoltaik/vorlesung/ss2014/unit4/optical_properties_silicon.pdf (accessed on 13 January 2017). 
28. Dao, V.T.S.; Shimonomura, K.; Kamakura, Y.; Etoh, T.G. Simulation analysis of a backside-illuminated multi-collection-gate image sensor. ITE Trans. Media Technol. Appl. 2014, 2, 114-122. [CrossRef]

29. Wikipedia: Angular Resolution (Explanation). Available online: https://en.wikipedia.org/wiki/Angular_ resolution (accessed on 13 January 2017).

30. The Rayleigh Criterion. Available online: http://hyperphysics.phy-astr.gsu.edu/hbase/phyopt/Raylei. html\#c1 (accessed on 13 January 2017).

31. Kunikiyo, T.; Takenaka, M.; Kamakura, Y.; Yamaji, M.; Mizuno, H.; Morifuji, M.; Taniguchi, K.; Hamaguchi, C. A Monte Carlo simulation of anisotropic electron transport in silicon including full band structure and anisotropic impact ionization model. J. Appl. Phys. 1994, 75, 297. [CrossRef]

32. Canali, C.; Jacobini, C.; Nava, F.; Ottavini, G.; Quaranta, A.A. Electron drift velocity in silicon. Phys. Rev. B 1975, 12, 297-312. [CrossRef]

33. Canali, C.; Jacobini, C.; Ottavini, G.; Quaranta, A.A. Highfield diffusion of electrons in silicon. Appl. Phys. Lett. 1975, 27, 278. [CrossRef]

34. Sze, M. Physics and Properties of Semiconductors-A Review. In Physics of Semiconductor Devices, 3rd ed.; Wiley \& Sons: New York, NY, USA, 2007; pp. 62-69.

35. Temperature Dependence of Silicon Absorption Length (Figure 2). Available online: http://snap.lbl.gov/ ccdweb/ccd_data.html (accessed on 13 January 2017).

(C) 2017 by the authors. Licensee MDPI, Basel, Switzerland. This article is an open access article distributed under the terms and conditions of the Creative Commons Attribution (CC BY) license (http:/ / creativecommons.org/licenses/by/4.0/). 